Disclosure of Interest: None declared

DOI: 10.1136/annrheumdis-2017-eular.3652

\section{THU0325 RELATIONSHIP OF THE INITIAL SYMPTOMS TO THE DIAGNOSIS DELAY AND POOR PROGNOSIS IN PATIENTS WITH TAKAYASU ARTERITIS}

K. Matsumoto, Y. Kaneko, K. Izumi, K. Yamaoka, T. Takeuchi. Internal Medicine, Division of Rheumatology, Keio University School of Medicine, Tokyo, Japan

Background: Clinical courses of Takayasu arteritis are of great variety. Its non-specific initial symptoms can cause the delay in diagnosis and lead to poor prognosis. However, the symptoms of very early phase of Takayasu arteritis and their effects on diagnosis delay and prognosis are unclear.

Objectives: To clarify the characteristics of initial symptoms of Takayasu arteritis, the delay in diagnosis, and its relationship with prognosis.

Methods: The consecutive patients with Takayasu arteritis with analysable information in our hospital were enrolled. Initial symptoms, laboratory findings before diagnosis, the duration from symptom onset to diagnosis, and prognosis were investigated. Initial symptoms were divided into 7 groups; cranial symptoms (dizziness, syncope, headache, neck pain, hemi-paralysis, and jaw claudication), visual symptoms (vision loss and visual field loss), extremities symptoms (claudication of extremities, coldness of limbs, bilateral difference in blood pressure, and limb numbness), cardiac/aortic symptoms (dyspnea on exertion, palpitation, and chest compression), hypertension, general illness (fever, fatigue, body weight loss, and arthralgia), and abnormal medical examinations (heart murmur, bruit on any extremities, and abnormal chest X-ray). Poor outcome was defined as a cardiovascular surgery or death.

Results: A total of 98 patients were enrolled with the median observation period of 12.1 years (range, 1 month to 59 years). Eighty-seven $(88.7 \%)$ were female and the mean age at diagnosis was 37 years. The mean duration from the initial symptoms to diagnosis was 600 days. Thirty-four (34.7\%) patients had poor outcomes. The initial symptoms before diagnosis were cranial symptoms in $25 \%$, visual symptoms in $5 \%$, extremities symptoms in $20 \%$, cardiac/aortic symptoms in $7 \%$, hypertension in $9 \%$, general illness in $26 \%$, and abnormal medical examinations in $8 \%$. The duration from symptom onset to diagnosis was $792,52,567,752,1318,293$ and 1014 days $(p=0.10)$, respectively; the rate of poor outcome was $28,25,38,86,75,22$, and $25 \%(\mathrm{p}=0.010)$, respectively. The duration from symptom onset to diagnosis was longer in the patients with poor prognosis than those without ( 837 vs 493 days, $p=0.06$ ). The patients with extremities symptoms were younger ( 29.6 vs 38.9 years, $p=0.026$ ) than those with the other symptoms, and patients with cardiac/aortic symptoms were older (49.7 vs 36.3 years, $p=0.044)$. The patients without general illness showed lower levels of C-reactive protein ( 3.26 vs $5.86 \mathrm{mg} / \mathrm{dl}, \mathrm{p}=0.048$ ), erythrocyte sedimentation rate $(38.3$ vs $66.6 \mathrm{~mm} / \mathrm{h}, \mathrm{p}=0.01)$, and platelet counts $\left(29.4^{*} 10^{4}\right.$ vs $34.0^{*} 10^{4} / \mu \mathrm{l}$, $\mathrm{p}=0.09$ ), and resulted in poor outcome more frequently than those with (42.6 vs $22.6 \%, p=0.058$ ).

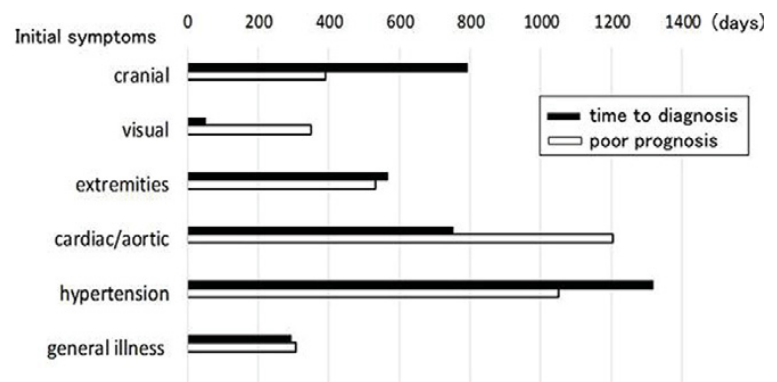

abnormal examinations

$\begin{array}{llllllllllll}0 & 10 & 20 & 30 & 40 & 50 & 60 & 70 & 80 & 90 & 100 & (\%)\end{array}$

Conclusions: The initial symptoms of Takayasu arteritis before diagnosis varied widely, and majority of them were non-specific. Lacking inflammatory signs were related with delayed diagnosis and poor prognosis.

References:

[1] Takayasu M, et al. Acta Soc Ophtalmol Jpn 1908; 12:554-561.

[2] Ishikawa K, et al. Circulation 1994; 90:1855-1860.

[3] Nakaoka Y, et al. Int Heart J 2013; 54:405-411.

[4] Vanoli M, et al. Arthritis Rheum 2005 15; 53(1):100-107.

Acknowledgements: We thank all the members of the Division of Rheumatology. Disclosure of Interest: None declared

DOI: 10.1136/annrheumdis-2017-eular.4181

\section{THU0326 SHORT AND LONG-TERM FOLLOW-UP WITH ADALIMUMAD IN REFRACTORY UVEITIS ASSOCIATED TO BEHÇET'S DISEASE. MULTICENTER STUDY OF 74 PATIENTS}

L. Domínguez-Casas $^{1}$, V. Calvo-Río ${ }^{1}$, E. Beltrán ${ }^{2}$, J. S-Bursón ${ }^{3}$, M. Mesquida ${ }^{4}$, A. Adán ${ }^{4}$, M. Hernandez ${ }^{4}, M$. H-Grafella ${ }^{5}$, E. V-Pascual ${ }^{6}$, L. M-Costa ${ }^{7}$,
A. Sellas ${ }^{8}$, M. Cordero-Coma ${ }^{9}$, M. Díaz-Llopis ${ }^{10}$, R. Gallego ${ }^{10}$, D. Salom ${ }^{10}$ J. G-Serrano ${ }^{11}$, N. Ortego ${ }^{12}$, J. Herreras ${ }^{13}$, A. G-Aparicio ${ }^{14}$, O. Maíz ${ }^{15}$, A. Blanco ${ }^{15}$, I. Torre ${ }^{16}$, D. Díaz-Valle ${ }^{17}$, E. Pato ${ }^{17}$, E. Aurrecoechea ${ }^{18}$, M. Caracuel ${ }^{19}$, F. Gamero 20 E. Minguez ${ }^{21}$, C. Carrasco 22, A. Olive ${ }^{23}$, J. Vázquez ${ }^{24}$, O. R-Moreno ${ }^{25}$, J. Manero ${ }^{25}$, S. Muñoz ${ }^{26}$, M. Gandia $^{27}$, E. Rubio-Romero ${ }^{28}$, F. Toyos-SMiera ${ }^{29}$, F. López-Longo ${ }^{30}$, J. Nolla ${ }^{31}$, M. Revenga ${ }^{32}, \mathrm{~N}$. Vegas-Revenga ${ }^{1}, \mathrm{C}$. Fernández-Díaz ${ }^{1}, \mathrm{R}$. Demetrio-Pablo ${ }^{1}$, M. González-Gay ${ }^{1}$, R. Blanco ${ }^{1} .{ }^{1} H U M V$, Santander; ${ }^{2} \mathrm{H}$ Mar, Barcelona; ${ }^{3} \mathrm{H}$ Valme, Sevilla; ${ }^{4} \mathrm{H}$ Clinic, Barcelona; ${ }^{5} \mathrm{H}$ General; ${ }^{6} \mathrm{H}$, Valencia: ${ }^{7} \mathrm{H}$ Peset, Zaragoza; ${ }^{8} \mathrm{H}$ Vall d'Hebron, Barcelona; ${ }^{9} \mathrm{H}$, León; ${ }^{10} \mathrm{H} \mathrm{FE}$, Valencia; ${ }^{11} \mathrm{H}$ Cecilio; ${ }^{12} \mathrm{H}$ Cecilio, Granada; ${ }^{13} \mathrm{IOBA}$, Valladolid; ${ }^{14} \mathrm{H}$, Toledo; ${ }^{15} \mathrm{H}$, Donostia; ${ }^{16} \mathrm{H}$ Basurto, Bilbao; ${ }^{17} \mathrm{HS}$ Carlos, Madrid; ${ }^{18} \mathrm{H}$, Torrelavega; ${ }^{19} \mathrm{H}$, Córdoba; ${ }^{20} \mathrm{H}$, Cáceres; ${ }^{21} \mathrm{H}$ Clínico, Zaragoza; ${ }^{22} \mathrm{H}$, Mérida; ${ }^{23} \mathrm{H}$ G Trias, Badalona; ${ }^{24} \mathrm{H}$, Ferrol; ${ }^{25} \mathrm{H}$ Servet, Zaragoza; ${ }^{26} \mathrm{HU}$ Infanta Sofía, San Sebastian de los Reyes; ${ }^{27} \mathrm{H} \mathrm{P}$ Mar, Cádiz; ${ }^{28} \mathrm{H}$ V Rocío; ${ }^{29} \mathrm{H}$ V Macarena, Sevilla; ${ }^{30} \mathrm{H}$ G Marañon, Madrid; ${ }^{31} \mathrm{H}$ Bellvitge, Barcelona; ${ }^{32}$ H R Cajal, Madrid, Spain

Objectives: To evaluate the efficacy of adalimumab (ADA) in short and long term follow-up in refractory uveitis of Behçet's disease (BD)

Methods: Multicenter study. Ocular inflammation was evaluated according to "SUN working Group" (Am J Ophthalmol 2005;140:509-516), and the macular thickening with OCT. A comparison was carried out between baseline, and follow-up visits. Results are expressed as mean $\pm S D$ or median [IQR]. Continuous variables were compared with Wilcoxon test.

Results: We studied 74 patients/132 affected eyes (39M/35W); mean age 38.7 \pm 11.3 . The ocular pattern was panuveitis $(n=45)$, posterior uveitis $(n=14)$, anterior uveitis $(n=14)$ and intermediate uveitis $(n=1)$. Before ADA, systemic treatment with corticosteroids, iv metilprednisolone $(n=23)$, Cyclosporin $A(58)$, azathioprine (33), metotrexate (31) and other drugs (28) was used. The dose of ADA was $40 \mathrm{mg} / 2$ weeks/ sc in monotherapy $(n=22)$ or combined $(n=52)$. Most patients showed a rapid and progressive improvement (TABLE). The 24 patients (37 affected eyes) with CME showed a significant improvement.

ADA was optimized in $23(31.1 \%)$ that were in remission for $15.3 \pm 9$ months Interval of administration was increased to $3(n=6), 4(13), 5(1), 6$ (1) and 8 weeks. After a mean follow-up of $13.0 \pm 9.7$ months after optimization, 21 patients were stable and 2 had a severe flare. In 4 patients ADA was stopped after $35.2 \pm 9.3$ months in remission. The main adverse effects observed were lymphoma $(n=1)$, pneumonia (1), and $2^{\circ}$ bacteriemia by E. Coli (1)

\begin{tabular}{|c|c|c|c|c|c|c|c|}
\hline & Basal & $2^{m}$ week & $1^{\text {t"}}$ month & $6^{\mathrm{m}}$ month & $1^{\text {tI year }}$ & $2^{m}$ year & $3^{10}$ year \\
\hline VA (mean $\pm S D)$ & $\begin{array}{l}0.5 \pm 03 \\
n=146\end{array}$ & $\begin{array}{l}0.6 \pm 0.3^{*} \\
n=144\end{array}$ & $\begin{array}{l}0.6 \pm 0.2^{*} \\
n=143\end{array}$ & $\begin{array}{l}0.7 \pm 0.2^{*} \\
n=120\end{array}$ & $\begin{array}{l}0.8 \pm 0.2^{*} \\
n=112\end{array}$ & $\begin{array}{l}0.8 \pm 0.2^{*} \\
n=89\end{array}$ & $\begin{array}{l}0.8 \pm 0.2^{*} \\
n=41\end{array}$ \\
\hline $\begin{array}{l}\text { Cells in the } \\
\text { anterior } \\
\text { chamber } \\
\text { (median [IQR]) }\end{array}$ & $\begin{array}{l}1[0-2] \\
n=144\end{array}$ & $\begin{array}{l}0[0-2]^{*} \\
n=142\end{array}$ & $\begin{array}{l}0[0-1]^{*} \\
n=142\end{array}$ & {$\left[\begin{array}{l}0[0-0]^{*} \\
n=120\end{array}\right.$} & $\begin{array}{l}0[0-0]^{*} \\
n=116\end{array}$ & $\begin{array}{l}0[0-0]^{*} \\
n=92\end{array}$ & $\begin{array}{l}0[0-0]^{*} \\
n=42\end{array}$ \\
\hline $\begin{array}{l}\text { Vitritis (median } \\
\text { [IQR]) }\end{array}$ & $\begin{array}{l}1[0-2] \\
n=145\end{array}$ & $\begin{array}{l}1[0-1]^{*} \\
n=143\end{array}$ & $\begin{array}{l}0[0-1]^{*} \\
n=143\end{array}$ & $\begin{array}{l}0[0-0]^{x} \\
n=124\end{array}$ & {$\left[\begin{array}{l}0[0-0]^{*} \\
n=116\end{array}\right.$} & $\begin{array}{l}0[0-0]^{*} \\
n=92\end{array}$ & $\begin{array}{l}0[0-0]^{*} \\
n=42\end{array}$ \\
\hline $\begin{array}{l}\text { Retinal } \\
\text { vasculitis (\% } \\
\text { affected eyes) }\end{array}$ & $\begin{array}{l}53.3 \% \\
n=144\end{array}$ & $\begin{array}{l}43.2 \% * * \\
n=141\end{array}$ & $\begin{array}{l}36.4 \%^{*} \\
n=142\end{array}$ & $\begin{array}{l}11.4 \%^{*} \\
n=123\end{array}$ & $\begin{array}{l}4.1 \% \%^{*} \\
n=116\end{array}$ & $\begin{array}{l}0.6 \%{ }^{*} \\
n=92\end{array}$ & $\begin{array}{l}0.6 \% *^{*} \\
n=42\end{array}$ \\
\hline $\begin{array}{l}\text { OCT }(\mu) \\
\text { (mean } \pm S D)\end{array}$ & $\begin{array}{l}345.5 \pm 134.5 \\
n=82\end{array}$ & $\begin{array}{l}341.1 \pm 108.6^{*} \\
n=65\end{array}$ & $\begin{array}{l}309.8 \pm 8^{*} \\
n=64\end{array}$ & $\begin{array}{l}268.1 \pm 38.4^{*} \\
n=52\end{array}$ & $\begin{array}{l}254.4 \pm 38.5^{*} \\
n=55\end{array}$ & $\begin{array}{l}259.5 \pm 41.2^{*} \\
n=42\end{array}$ & $\begin{array}{l}254.5 \pm 46.9^{\prime} \\
n=20\end{array}$ \\
\hline
\end{tabular}

Conclusions: ADA was effective in short and long-term follow-up in refractory uveitis associated to BD. Optimization or even suspension of ADA is possible. Disclosure of Interest: None declared

DOI: 10.1136/annrheumdis-2017-eular.3577

\section{THU0327 ASSOCIATION BETWEEN THE TC/HDL RATIO AND DISEASE ACTIVITY IN PATIENTS WITH TAKAYASU ARTERITIS}

L. Pan ${ }^{1}$, J. Du ${ }^{2}$, H. Liao ${ }^{1} .{ }^{1}$ Rheumatology and Immunology; ${ }^{2}$ Beijing An Zhen Hospital, Capital Medical University., Beijing, China

Background: Accelerated atherosclerosis has become the main cause of morbidity in patients with autoimmune diseases such as RA and SLE [1]. The Cholesterol/High-density Lipoprotein Cholesterol (TC/HDL-C) ratio is a high discriminatory power index for coronary heart disease. A high TC/HDL-C ratio has been intensively used as a predictor of CVDs [2]. EULAR Task Force recommended that the TC/HDL-C ratio should be regarded as an important prognostic indicator for future cardiovascular disease (CVD) in patients with rheumatoid arthritis (RA) [3]. However, the relationship between the TC/HDL-C ratio and disease activity of Takayasu arteritis (TAK) is unclear.

Objectives: To investigate changes in the TC/HDL-C ratio and to evaluate the relationship between the TC/HDL-C ratio and disease activity of TAK.

Methods: A retrospective study of 103 patients with TAK and 73 healthy controls was performed. We compared the triglyceride (TG), TC, low-density lipoprotein cholesterol (LDL-C), HDL-C and TC/HDL-C ratio between patients and healthy controls, and we analyzed correlations between the lipid parameters and indexes 